\title{
Relationship Between Autism And Nasal Cytology
}

\author{
Arturo Armone Caruso ${ }^{1}$,Simonetta Masieri ${ }^{2}$,Carlo Cavaliere ${ }^{2}$,Josephine \\ Salzano ${ }^{1}$,Maria Rosaria Salzano ${ }^{3}$,Lina Cogliani ${ }^{3}$, Silvana Figlioli ${ }^{3}$, \\ Salvatore Del Prete ${ }^{4}$, Lydia Ferrara ${ }^{5}$ \\ ${ }^{l}$ Otc Aias Of Afragola, District Leutrec ;Napoli,Italy \\ ${ }^{2}$ Policlinico Umberto $I^{\circ}$ Università "Sapienza "Roma \\ ${ }^{3}$ Tpnee Aias Of Afragola District Leutrec ;Napoli,Italy \\ ${ }^{4}$ Department Of Head And Neck. University Of Naples Federico Ii, Naples, Italy \\ ${ }^{5}$ università Di Napoli, Italy
}

\begin{abstract}
Autism is a complex psychopathology caused by an abnormal neuropsychological development to which an individual is not able to self-regulate their feelings towards a coherent and correct psychomotor activity and communicative.Many factors contribute to the onset of this disease, and recent studies have highlighted, which important causes, in addition to the neuro-immunological interactions, the increased presence in the blood of inflammatory substances such as cytokines. Our research has been devoted to the identification of inflammatory cells in the nose in autistic patients by panoptic staining and microscopic observation. From the results obtained it proved the presence of a greater number of inflammatory cells in autistic subjects compared to normal individuals.
\end{abstract}

Keywords: Autism, Nasal Citology, Immunity, Allergy

\section{Introduction}

Autism spectrum disorder (ASD) is a behavioral syndrome caused by a developmental disorder biologically determined, with onset in the first three years of life. The areas mainly affected are those of reciprocal social interaction, the ability to communicate ideas and feelings and the ability to establish relationships with others [1] [2] [3]. Autism, therefore, appears as a "permanent" disability that accompanies the subject in its life cycle, even if the characteristics of the social deficit assume variable expressivity in time. We do not know exactly what the cause of this disease, but it is assumed that environmental factors, immunological and neurological, play an essential role in the development of ASD. Recently, research has focused on the links between the immune system and the nervous system, including their possible role in the development of ASD [4]. These Neuroimmunologic interactions begin during embryogenesis and persist throughout the life of an individual until full neurological development, through a balanced and normal immune response. Immunological abnormalities reported in autistic children, however, are characterized by a poorly regulated response concerning an abnormal cell of HT1 type, abnormal ratio $\mathrm{T}(\mathrm{H}) 1)$ / $\mathrm{T}(\mathrm{H}) 2$, altered levels of cytokines, lack of lymphocytes, decreased cleavage response T1 cells, the levels of serum immunoglobulins imbalance.

The immune system has a fundamental role to defend the body against harmful external agents attacks for our body and consists of an innate and an acquired component. The innate component is the first line of defense that causes an immediate and widespread response against pathogens by certain types of cells such as the macrophages, which are able to recognize the bacteria; the granulocytes, which recognize bacteria and parasites; the cellulle dendritic, which recognize the virus, the killer cells, which recognize tumor cells; the mast cells, which are responsible for the allergic response.

The action of macrophages against bacteria manifests itself not only through the release of substances such as cytokines and chemokines, which push the granulocytes to migrate to the site of infection and attack the invader, causing the typical phenomena of inflammation, but also urging the intervention of acquired immune cells, the lymphocytes. Two types of lymphocytes are known: B and T lymphocytes which play precise and targeted attacks against intruders: B cells produce antibodies against foreign agents and act outside of cells, $\mathrm{T}$ cells act against the aggressors within the cells. The $\mathrm{T}$ cells are divided into two types: cytotoxic $\mathrm{T}$ lymphocytes that directly attack the aggressor; helper $\mathrm{T}$ cells that stimulate the attack on the part of B lymphocytes and macrophages.

The $\mathrm{T}$ helper (Th) are divided into two Th1 and Th2. phenotypes that in healthy individuals are in perfect balance with each other. The increase of Th2 cells chronically suppresses the possibility of an effective immune response against the tumor or the virus; an increase of them occurs in patients with allergic diseases. Patients with autoimmune diseases have an imbalance in favor of Th1 cells that determine a chronic inflammatory condition that destroys the body's tissue. Vargas and colleagues using different techniques showed that some of the cells involved in the innate inflammatory response of the brain, astrocytes and microglia, were 
increased pathologically in autistic subjects. These cells were activated by a high presence of cytokines in the cerebrospinal fluid [5]

A role of primary importance are the maternal inflammatory processes during pregnancy: many epidemiological data have consistently revealed a strong correlation between prenatal exposure to pathogens and the onset of autism spectrum disorders in children. Studies in the rats have shown that activation of the maternal immune system during pregnancy exposing the developing fetus to specific chemical mediators of immunity maternal, it is resulted in a significant neurological impairment, immunological and behavioral in the offspring of these animals, comparable to immune disorders and neurological and behavioral abnormalities verified in some autistic patients [6]. Moreover autism has been linked with the autoimmunity and an association between genes and innate- immunity, including the human leukocyte antigen (HLA) - DRB1: there is the possibility that the immune activity is so abhorrent during the period neuro--developmental to cause neurological dysfunction of ASD [7].

Based on the above and some of our conjectures we started in 2000 to assess by physical examination of patients with ASD some clinical features compatible with immunological factors [8][9][10].Among these elements is the one that we most obvious result, both for rapid diagnosis, both for immediate evaluation, is the framework rhinitis. For this reason we proceeded to the cytological diagnosis of these events and we have tried to document a relationship between framework inflammation of the nose of people with ASD, characterized by a greater presence of inflammatory cells, compared to individuals of the same age and sex, no autistics. Also this type of research was also made based on the institutional purposes of Afragola AIAS (Italian Association of Disadvantaged Assistance Afragola). The moral authority structure nonprofit, was established in 1985. In 2002 he was legally recognized by the Campania Region and registered in the Register of Legal Persons to n. 56 of $16 / 05 / 2002$. In 2001 he was recognized association of promotion of peace and human rights of the Region Campania and registered under no. 1695. It is a center of Accredited Rehabilitation at the Campania Region. Its main activities are the search for those strategies that can contribute to improving the lives of people with disabilities.

\section{Materials And Methods}

At the center of AIAS Afragola, they were selected 150 children of both sexes ( 85 males; 65 females) (mean age, 5.1 years min 3, maximum 10, in a period from 2005 to 2015). The aim was to document the presence of inflammatory cells (neutrophils, eosinophils, mast cells and lymphocytes) in patients with ASD and to relate these results with a homogeneous group of entities exempt from this disease. They excluded patients with infectious diseases and oncology. As statistically significant value was considered to $\mathrm{P}<0.05$, and the Student's test was applied. The data collection was carried out by performing a summation of the observed cells and calculated as a percentage of all the other cells observed.

Particular importance in the study was paid to sampling times and processing cytology. The cytological was carried out, after the obligations on privacy, informed consent and medical history, by means of scraping technique, crawling 2-3 times a rinosonda (Nasal-scraping ${ }^{\circledR}$ ) on the mucosal surface of the central zone the inferior turbinate. A sterile swab to urethra, soaked in sterile saline and then crawled on the middle part of the inferior turbinate in children below the age of five was used.

The nasal mucosal cells were placed on an electrostatically charged cytology slide (Super Frost Plus Menzel - Gläser, Thermo Scientific, Milan, Italy). The cells were then stained according to the panoptic Pappenheim method (3 min in pure May-Grunwald dye [Carlo Erba, Milan, Italy],6 min. in 50\% May-Grunwald dye; $1 \mathrm{~min}$. in bidistilled water [Carlo Erba, Milan, Italy]; and $30 \mathrm{~min}$. in Giemsa solution [Carlo Erba, Milan, Italy] diluted 1:10 v/v). The slide was then covered with a \# 1 cover glass with dimensions of $24 \times 50 \mathrm{~mm}$ and observed under an optical microscope (Nikon Eclipse 200) at $100 \mathrm{x}$ enlargement in oil-immersion.

\section{Results And Discussion}

The motivation for this work was to identify a possible correlation between the ASD and immune system disorder. For this reason, it was chosen the nasal mucosa, which for its cellular structure is easier to observe in inflammatory conditions [11]. The study of the nasal mucosa has highlighted a very important factor in the evaluation of the examined subjects, who had no allergies nor from a point anmnestico, nor instrumental: it is seen, in fact, that in subjects suffering from ASD is present a higher concentration of inflammatory cells (neutrophils, eosinophils, mast cells and lymphocytes)[ Fig 1]. They were excluded values of neutrophils, because such cells may also be present in the absence of immunologically active conditions, since their function is also to be "scavenger" cells and can not always be subtle problem in the diagnosis of a disease of immunological origin [12]. In normal subjects, also, the presence of inflammatory cells may be related to a form of cell rhinitis (NARES, NERESMA, NARMA) nature of likely non-allergic or allergic of a frame not yet completely developed given the age of some subjects . This correlation is in agreement with the international 
literature that he theorize a relationship between the immune system and ASD [7] [ 13].More research is needed to explain how these immune abnormalities arise and how they can affect the neurological development, behavior and blended learning in the short and long term, and to indicate how it is possible to combat the main autism pathological processes.

\section{Conclusions}

The results show a higher concentration of inflammatory cells in patients with ASD and the method we use for simple running, with consistent and repeatable results, it allows quickly immunological evaluation of the subjects examined and given the low cost could shed more light diagnostic of the disorder, and consequently implementing new therapeutic frontiers associated with the rehabilitation program

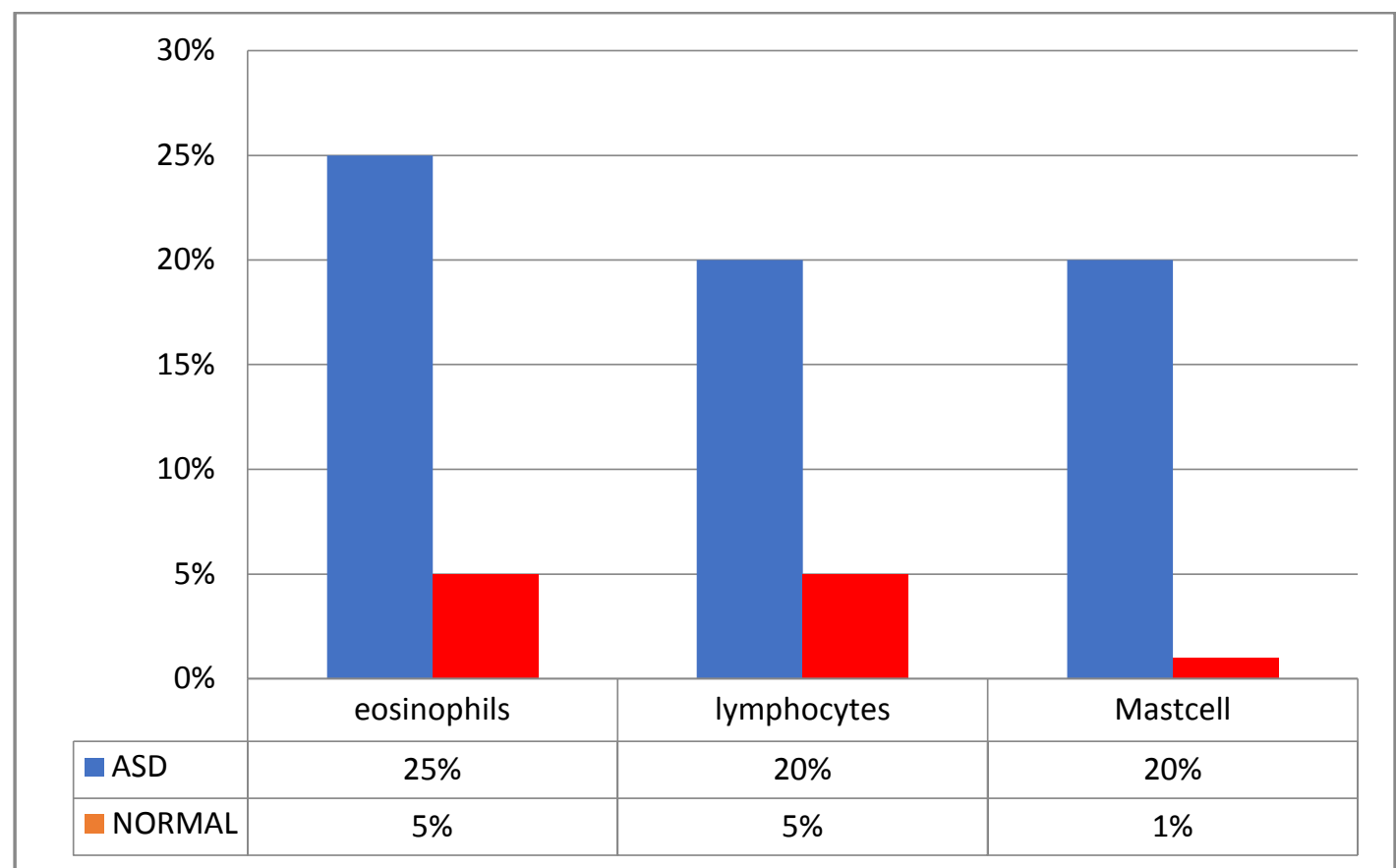

Figure 1: Percentage relationship between subjects with ASD and normal subjects

\section{References}

[1]. G. Baird, H.Cass,V.Slonim Diagnosis of autisms BMJ 327(7413),2003,488-93

[2]. T.P.Berney Autism an evolving concept. Br J Psychiatry. 176, 2000, 20-25

[3]. P. Szatmari The causes of autism spectrum disorders. BMJ. 326(7382), 2003,173-4

[4]. A.Meltzer, J.Van de Water The Role of the Immune System in Autism Spectrum Disorder. Neuropsychopharmacology.42(1), 2017, 284-298.

[5]. DL Vargas, C.Nascimbene, C.Krishnan, AW Zimmerman, CA Pardo Neuroglial Activation and Neuroinflammation in the Brain of Patients with Autism. Ann Neurol 2005,67-81

[6]. Gloria B. Choi, Yeong S. Yim, Helen Wong, Sangdoo Kim, Hyunju Kim, et al. The maternal interleukin-17a pathway in mice promotes autism like phenotypes in offspring Science AAAS 351(6276),2016, 1-12

[7]. P.Ashwood, S.Wills, J.Van de Water The immune response in autism: a new frontier for autism research. J Leukoc Biol. $80(1), 2006,1-15$

[8]. A.Armone Caruso Lo studio della mucosa nasale nei bambini autistici: aspetti citologici e morfologici 2nd National Conference on Neurobiology of Autism : New therapeutic advances and educational courses Teramo, 28 - 29 May 2011

[9]. L. Ferrara, D. Naviglio, A. Armone Caruso Phytoterapy treatment of allergic rhinitis in autistic subjects, European Scientific Journal 3, 2014 1-6

[10]. L. Ferrara, A. Armone Caruso, D. Naviglio, M. Gallo Phytotherapic Formulation for Allergic Rhinitis in Autistic Children Advances in Research 2(8), 2014,441-454

[11]. M. Gelardi, Atlante di citologia nasale per la diagnosi differenziale delle rinopatie, Edi. Ermes, Milano 2012

[12]. H.Jyonouchi Autism spectrum disorders and allergy: observation from a pediatric allergy/immunology clinic. Expert Rev Clin Immunol. 6(3), 2010, 397-411.

[13]. T.C. Theoharides, Autism spectrum disorders and mastocytosis, Int J Immunopathol Pharmacol 22,2009, 859-865. 